\title{
Designing Smart Sewerbot for the Identification of Sewer Defects and Blockages
}

\author{
Ghulam E Mustafa Abro ${ }^{1}$, Bazgha Jabeen ${ }^{2}$, Ajodhia $^{3}$, Kundan Kumar ${ }^{4}$, Abdul Rauf ${ }^{5}$, Ali Noman ${ }^{6}$, Syed Faiz ul \\ Huda $^{7}$, Amjad AliQureshi ${ }^{8}$ \\ Department of Electronic Engineering ${ }^{1,2,8}$, Computer Science ${ }^{3}$, Electrical Engineering ${ }^{4,5,6,7}$ \\ Hamdard University, Karachi, Sindh Pakistan 1,2,3 \\ Indus University, Karachi Sindh Pakistan ${ }^{3,6,7}$ \\ Benazir Bhutto Shaheed University of Technology \& Skill Development, Khairpurmirs, Pakistan ${ }^{4}$ \\ National University of Computer and Emerging sciences NUCES FAST, Karachi Pakistan ${ }^{5}$ \\ Sir Syed University of Engineering and Technology SSUET, Karachi Pakistan ${ }^{8}$
}

\begin{abstract}
Internet of thing (IoT) is a new concept where the term 'thing' is associated with the configurable sensors and devices no matter domestic or industrial, whereas bridging up a relationship in between these things and internet protocol is known as Internet of thing. Moreover, the same concept has been introduced in the field of robotics as 'Internet of Robotic Things (IoRT)', which is mainly concerned with active sensorization of sensors dully interfaced with any type of robots i.e. autonomous unmanned ground vehicle (UGV). This paper describes the prototyping of an autonomous sewerbot that will not only identify the sewer defects in sewerage pipelines but will also identify the type of blockages using the technique of digital image processing. Furthermore, the deployed configurable sensors will also share the attributes of particular sewerage line on IoT such that temperature, humidity, availability of hazardous gases, exact depth at which it is available and global positioning using GPS module. The paper also provides the brief construction of this mechatronic and amphibian system via which it can extricate the blockages from sewerage lines along with wireless camera surveillance.
\end{abstract}

Keywords-Internet of Robotic Things (IoRT); GPS; humidity; internet protocol; temperature; wireless communication and sewer defects

\section{INTRODUCTION}

There are some problems that are occurring mostly in various countries of the world whether developing or already developed one. Sewer pipeline problem is one of them in which the main sewerage line has been mostly encountered with severe problem of blockage or leakage. This results the effusion of filthy water into roads and streets of our city and thus producing traffic congestion and directly harm the people who used to travel through that particular road or street. Moreover, if same problem occurs in the apartments then all residents of the building will suffer from non-hygienic environment. The paper categorizes these problems into six major defect types as illustrated in Fig. 1 and are mentioned as:

- Pipe tree root issues

- Cracked pipeline issues

- Sewerage blocking issues
- Pipe corrosion and deterioration issues

- Pipeline alignment issues (Bellied pipe) and

- Pipe leakage issues

Before going further, one must understand the main causes for the above-mentioned issues. Discussing the pipe tree root issue, it is one of the problem in which the tree root material extends itself from the skeleton of the pipeline and hence creates a gap from which the water may start escaping out. Sometimes the sewerage pipelines are also cracked due to variation in local temperature conditions of the environment, this is known as cracking issue of sewerage lines. Moreover, one of the most rapidly occurring problems is the accumulation of drain water in sewerage pipelines. It is because of the several materials that are non-intentionally come in pipeline and due to their larger mass or area; they block the way out for drain water. In such circumstance, one may pursue any chemical to clear the pipeline and if the used pipeline is other than the poly vinyl chloride (PVC) material it would be deteriorated and are de-shaped easily such problem is known as pipe corrosion and deterioration issues. In some of the places, it is observed that the angle attached within PVC pipes are not tightly attached with glue material and hence after sometime, leakage occurs in it and this is also one of the reasons due to which the drain water comes out of the sewerage pipelines. It is also studied that if the geological surface or foundation is misbalanced during construction and a pipeline is placed inside it. This pipeline will no longer stay there and will soon settle down and de-shaped itself again hence one may experience an inclination in pipe; such problem is known as Bellied pipeline issue. After studying these issues, the paper suggests internet of robotic things (IoRT) oriented solution. This research focuses on the prototype of an unmanned ground vehicle (UGV), specially designed on fiber material that can walk as well as swim inside the sewerage lines.

This water-proof mechatronic and (IoRT) oriented system has an ability to share the attributes of sewerage pipelines such that the temperature, availability of hazardous gases, exact altitude at which sewerbot is doing surveillance using wireless camera and exact global positioning using neo GPS module. 
In addition to this, it has been equipped with a specially designed rudder dully coupled with dc gear motor that will help sewerbot to clear the blockages. The paper shares the deployment of wireless waterproof $4 \mathrm{~K}$ camera that will provide images to base station for the identification of sewer defects using the techniques of digital image processing.

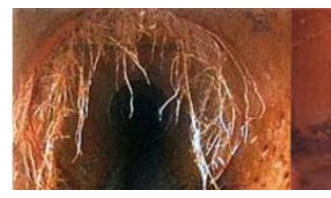

Tree root issue

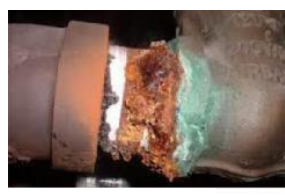

Corrosion \&

Deterioration

Fig. 1. Six Major Sewer Defects in Sewerage Lines.

\section{LITERATURE REVIEW}

There had been lot of solutions that had been proposed earlier and to check their pros. and cons. it is important to go through their brief methodology and techniques. There are several systems that identify the defects in underground pipelines using digital image processing techniques i.e. gradient filtering, thresholding, segmentation and histogram computation [1]. Whereas some of the proposed solutions were fully automated and designed to monitor the hazardous gases inside the sewerage lines [7]. One may find various research solutions on wireless exchange of data, mission accomplishment along with planning and localization using different micro-controllers [2]. Moreover, mainly such systems are proposed for the fields where human can never execute the task with maximum efficiency and such areas are classified as dull, dirty, difficult and dangerous. Here the proposed area is the sewerage lines that comprises of all such dimensions hence many robots have been suggested i.e. MAKRO series which have water-proof casing and have optical sensors to sense the hurdles arriving in front [2].

Moreover, one may find the manveurability of these control systems inside T, X and L shaped junctions and are driven using wireless medium of bluetooth. The addition of path generation and positioning make them one of their kinds [3]. One can see the structures are either driven in wet pipelines or in pipelines having filthy water at one time hence in this regard there are various waterproof and dirt resistive designs already been proposed such that KURT [3]. Majority of the proposed systems can run round and over obstacles via wireless instruction set but they are built for experimental purposes only [4]. Every autonomous wheeled mobile robot has its own limitations hence the pros. and cons. related to such systems are mentioned briefly in Table 1.
TABLE I. DIFFERENT SEWERBot TECHNIQUES

\begin{tabular}{|c|c|c|c|}
\hline $\begin{array}{l}\text { Sr. } \\
\#\end{array}$ & Techniques & Pros. & Cons \\
\hline 1 & $\begin{array}{l}\text { Image } \\
\text { Processing } \\
\text { based robot [1] }\end{array}$ & $\begin{array}{l}\text { It provides area, width, } \\
\text { length, radius, diameter, } \\
\text { roundness and centroid of } \\
\text { sewerage lines. }\end{array}$ & $\begin{array}{l}\text { It does not provide } \\
\text { any classification of } \\
\text { defects. }\end{array}$ \\
\hline 2 & MAKRO I [2] & $\begin{array}{l}\text { It is self-propelled with } \\
\text { TV camera. }\end{array}$ & $\begin{array}{l}\text { It was operated } \\
\text { through wired } \\
\text { technology and } \\
\text { unable to drive in } \\
\text { bended pipelines. It } \\
\text { had some external } \\
\text { disturbances too. }\end{array}$ \\
\hline 3 & $\begin{array}{l}\text { Articulated } \\
\text { MAKRO robot } \\
\text { [3] }\end{array}$ & $\begin{array}{l}\text { It has } 3 \text { degree of freedom } \\
\text { DOF and Opertable in a } \\
\text { Laokoon network easily. It } \\
\text { shares the attributes of the } \\
\text { field such that obstacle } \\
\text { detection, landmark } \\
\text { detection and a laser } \\
\text { pointer is used to extricate } \\
\text { blockages. }\end{array}$ & $\begin{array}{l}\text { It was controlled } \\
\text { through wire } \\
\text { whereas optical } \\
\text { sensors deform the } \\
\text { pipe lines and it was } \\
\text { not doing the } \\
\text { classification of the } \\
\text { defects. }\end{array}$ \\
\hline 4 & $\begin{array}{l}\text { KARO[4] \& } \\
\text { PIRAT [5] }\end{array}$ & $\begin{array}{l}\text { Battery operated and long } \\
\text { drive mode. It is } \\
\text { Incorporated with } \\
\text { configurable sensors that } \\
\text { shares the attributes and } \\
\text { stabilized using Fuzzy } \\
\text { logic based control design. }\end{array}$ & $\begin{array}{l}\text { Operator identifies } \\
\text { the defects whereas } \\
\text { the drive was slower } \\
\text { and unstable because } \\
\text { of mamdani scheme. }\end{array}$ \\
\hline 5 & $\begin{array}{l}\text { EURO-Robot } \\
\text { [6] }\end{array}$ & $\begin{array}{l}\text { It has been designed on } \\
\text { probabilistic robot } \\
\text { navigation scheme }\end{array}$ & $\begin{array}{l}\text { Partially observe the } \\
\text { environment }\end{array}$ \\
\hline 6 & $\begin{array}{l}\text { Sewersnort } \\
\text { robot [7] }\end{array}$ & $\begin{array}{l}\text { A low cost, unmanned, } \\
\text { fully automated UGV to } \\
\text { monitor hazardous } \\
\text { gases. It has an ability to } \\
\text { trace the location and } \\
\text { provides an accurate gas } \\
\text { exposure }\end{array}$ & $\begin{array}{l}\text { It has mainly focused } \\
\text { on the detection of } \\
\text { hazardous gases } \\
\text { inside the pipelines. }\end{array}$ \\
\hline 7 & Kantaro [8] & $\begin{array}{l}\text { It can work within number } \\
\text { of different bends and can } \\
\text { identifying nine different } \\
\text { types of faults from the } \\
\text { sensor data }\end{array}$ & $\begin{array}{l}\text { The feature of } \\
\text { Internet of things is } \\
\text { missing. }\end{array}$ \\
\hline 8 & $\begin{array}{l}\text { KA-TE } \\
\text { Systems [8] }\end{array}$ & $\begin{array}{l}\text { Provide better results in } \\
\text { terms of surveillance and } \\
\text { commercially available }\end{array}$ & $\begin{array}{l}\text { Classification of } \\
\text { sewer defects is } \\
\text { missing and does not } \\
\text { provide the detection } \\
\text { of gases \& } \\
\text { expensive. }\end{array}$ \\
\hline 9 & $\begin{array}{l}\text { Inspection tool } \\
\text { Pipe hunter [9] }\end{array}$ & $\begin{array}{l}\text { It provides best visuals in } \\
360 \text { degree }\end{array}$ & $\begin{array}{l}\text { It was of large size } \\
\text { and could not } \\
\text { provide results in } \\
\text { dark }\end{array}$ \\
\hline 10 & Versatrax [9] & $\begin{array}{l}\text { It is of different adjustable } \\
\text { length }\end{array}$ & $\begin{array}{l}\text { All were based on } \\
\text { wires. }\end{array}$ \\
\hline
\end{tabular}


There are several mobile robots other than this type that uses an extensive application of image processing. While measuring the land markings inside the pipelines some solution proposes to calculate the probabilistic errors through images [10]. After studying different object recognition algorithms one may find majority of them are based on edge detection [11] whereas texture based approaches and Gabor wavelets are also used in some of the research papers [12]. Various authors used stereo vision along with additional sensors for detecting curbs [13]. In the field of autonomous vehicles various research manuscript suggest path planning too with obstacle avoidance [13] which can be implemented in same way in sewerage pipelines too. For the active sensorization, various manipulator designs and robots are interfaced with Wi-Fi shields and ultimately sharing their attributes using dynamic internet protocol (IP) [14]. In addition to this, the papers also proposed the latency comparison in between conventional systems and IoT based systems [15].

\section{Methodology}

The paper suggests the detailed methodology for prototyping an IoRT based sewerbot that can even move in bended pipelines as well. Before initiating this prototype, it was very important to have its structure design hence in this regard solid works software is used. One can find the design with accurate measurements in Fig. 2.

Moreover, the proposed structure is waterproof and dirt whereas the components used in this sewerbot are as:

- DC gear motors

- Raspberry Pi Controller

- ESP8266

- Wireless 4K Camera

- DHT 11 (Humidity \& Temperature Sensor)

- BMP-180 (Pressure and altitude sensor)

- MQ-5 Gas Sensor

- GPS Neo Module

First, the user will generate an instruction key through IP connect app that is mostly available in today's smart phones and then this instruction will be received at raspberry pi through TCP/IP protocol and Wi-Fi. The raspberry pi will enable its manveurability mechanism to move further. It should be noted that keys are already assigned in programming for moving this prototype in forward, backward, right and left direction. Moreover, another key will be pressed to turn on the image surveillance and image processing using wireless camera. The image data set has been already stored in the memory of our proposed pi controller from which these captured images will be correlated and will share the exact fault or defect type in pipeline. If the camera detects any sort of blockage, its specially designed rudder mechanism will be turned on and may extricate the lines autonomously as illustrated in Fig. 4. While extricating the line the attributes such that availability of hazardous gases, temperature, humidity, pressure and exact depth will be sensed and dully communicated to user using internet protocol. This whole procedure can be seen executable by visualizing the block diagram as illustrated in Fig. 3.

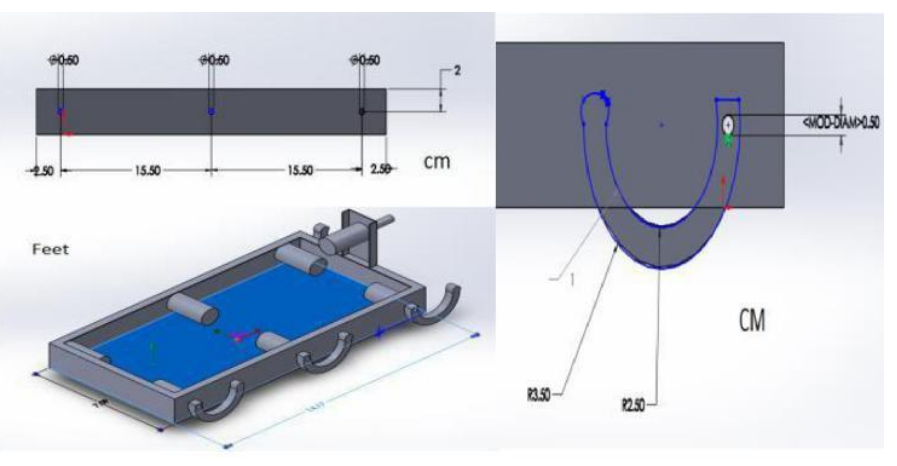

Fig. 2. Computer Aided Engineering Drawing of Sewerbot.
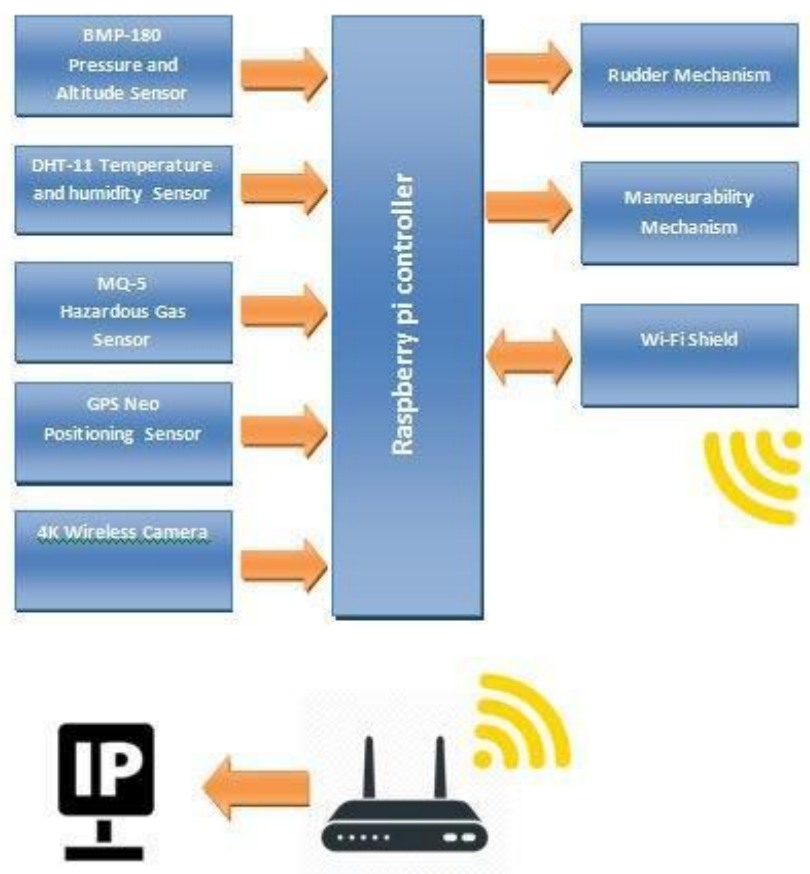

Fig. 3. Block Diagram of Sewerbot.

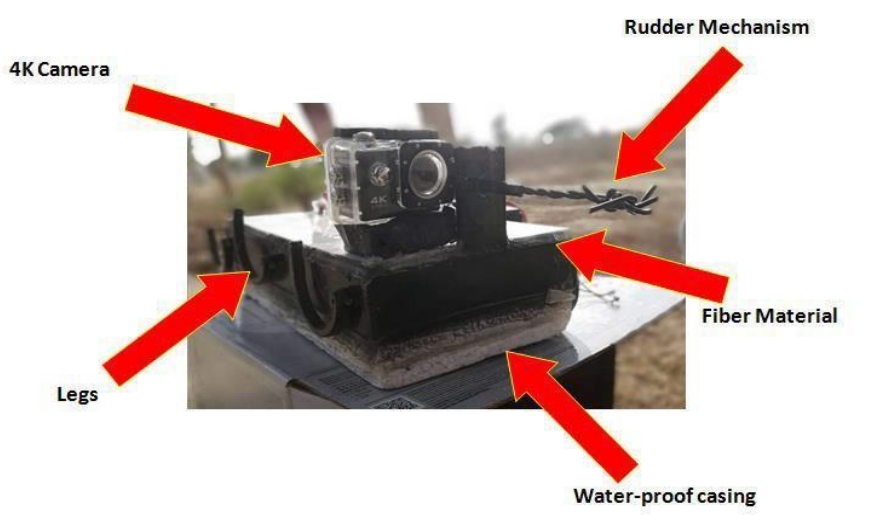

Fig. 4. Proposed Sewerbot with all Components. 


\section{RESUlts}

The proposed system has been made with the incorporation of above-mentioned electronic components and then it has been waterproofed using glue material dully melted and then this prototype has been dipped inside. The battery outlet was flexible that it can be opened and can be charged with 12 DC Volt charger. For demonstration work, the sample pipeline has been constructed and the polythene material has been blocked for extricating test as shown in Fig. 5.

The successful demonstration had been performed in the presence of water as well as wet environment. The proposed sewerbot concluded the results by clearing this line as illustrated in Fig. 6.

The visuals can be seen on TCP/IP application whereas the retrieved images captured by the wireless $4 \mathrm{~K}$ camera will be transmitted through file transfer protocol (FTP) to pi and then here the gradient and segmentation technique is use that provides below mentioned results in Fig. 7.
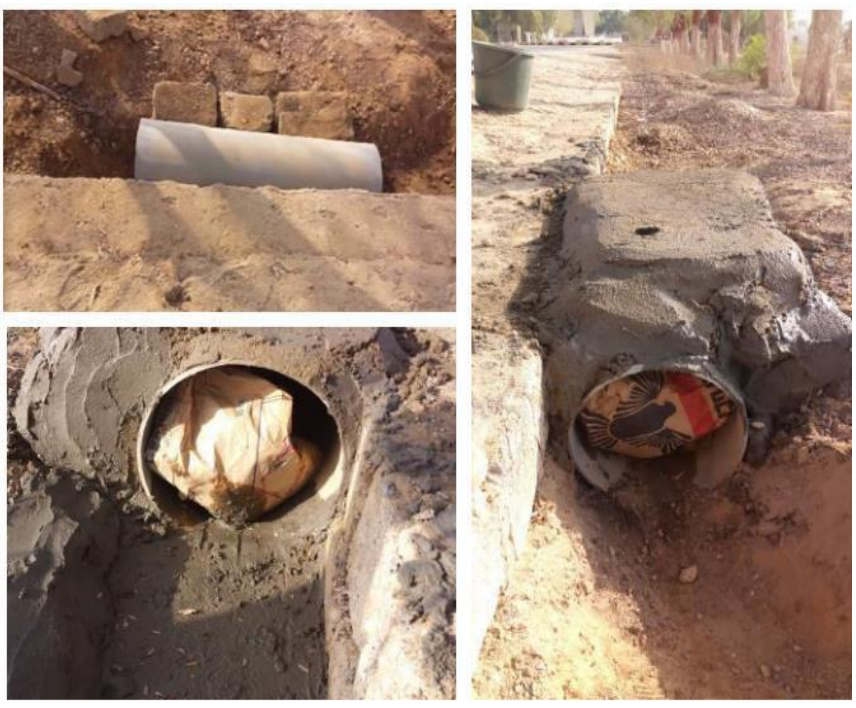

Fig. 5. Construction Site for Practical Implementation.
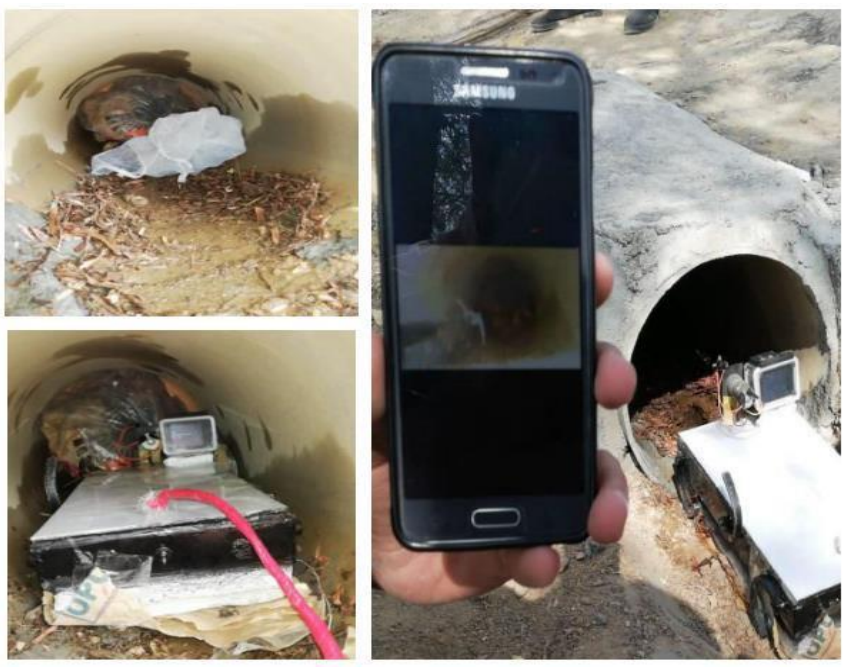

Fig. 6. Demonstration Work of Sewerbot In Sewerage Line.

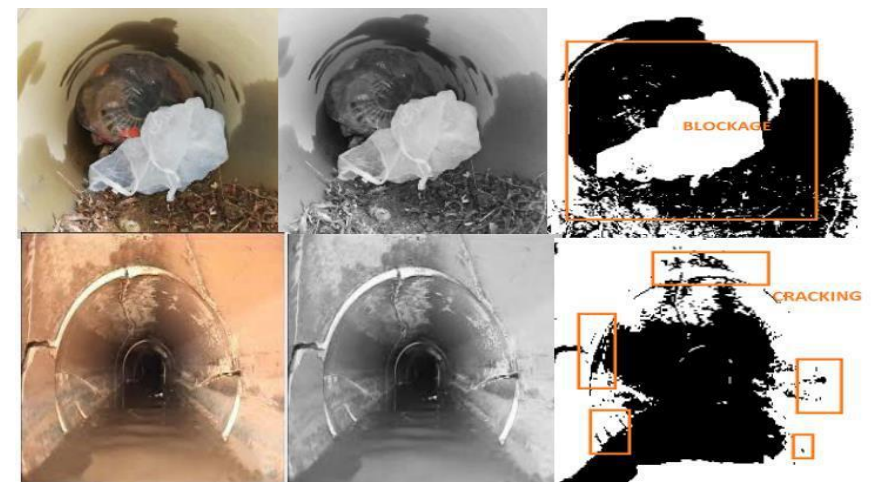

Fig. 7. Image Processing Sewer Defect Analysis.

\begin{tabular}{|c|c|c|}
\hline -1 Jazz 尺 & 4:12 PM & $84 \%$ \\
\hline & 10 & \\
\hline
\end{tabular}

Department of Electronic Engineering

\section{Parameters:}

\section{Temperature Value is 31 Celsius}

Humidity Value is $24 \%$

Hazardus Gas is Detected

Hurdle is Arrived

Altitude is 8 Feet

Fig. 8. Temperature, Humidity and Other Parameters Shared on IP.

Furthermore, this sewerbot will convey the necessary information of that particular sewerage line to user using internet protocol as shown in Fig. 8.

\section{CONCLUSION}

After detailed survey of all sewer robots and sewer defects the paper addresses the best solution comparatively solving the majority of the issues and with the induction of internet of robotic things (IoRT) this prototype becomes one of its kinds. Furthermore, this sewerbot not only survey and share the attributes of sewerage lines but autonomously identify the problem type inside the sewerage line.

\section{REFERENCES}

[1]. Osama, Moselhi, and Tariq Shehab-Eldeen. "Automated detection of surface defects in water and sewer images." Automation in Construction 8 (1999): 581-588.

[2]. Rome, Erich, Joachim Hertzberg, Frank Kirchner, Ulrich Licht, and Thomas Christaller. "Towards autonomous sewer robots: the MAKRO project." Urban Water 1, no. 1 (1999): 57-70.

[3]. Berns, Karsten, Thomas Christaller, Ruediger Dillmann, Joachim Hertzberg, Winfried Ilg, Manfred Kemmann, Erich Rome, and Heiner

[4]. Stapelfeldt. "LAOKOON - lernfaehige autonome kooperierende Kanalroboter." KI 11, no. 2 (1997): 28-32.

[5]. Kuntze, H. B., D. Schmidt, H. Haffner, and M. Loh. "KARO-A flexible robot for smart sensor-based sewer inspection." In Proc. Int. Conf. No Dig'95, Dresden, Germany, 19, pp. 367-374. 1995. 
[6]. Kirkham, Robin, Patrick D. Kearney, Kevin J. Rogers, and John Mashford. "PIRAT - a system for quantitative sewer pipe assessment." The International Journal of Robotics Research19, no. 11 (2000): 10331053.

[7]. Hertzberg, Joachim, and Frank Kirchner. "Landmark-based autonomous navigation in sewerage pipes." In Advanced Mobile Robot, 1996., Proceedings of the First Euromicro Workshop on, pp. 68-73. IEEE, 1996.

[8]. Kim, Jihyoung, Jung Soo Lim, Jonathan Friedman, Uichin Lee, Luiz Vieira, Diego Rosso, Mario Gerla, and Mani B. Srivastava. "Sewersnort: A drifting sensor for in-situ sewer gas monitoring." In Sensor, Mesh and Ad Hoc Communications and Networks, 2009. SECON'09. 6th Annual IEEE Communications Society Conference on, pp. 1-9. IEEE, 2009.

[9]. Nassiraei, A.A.F., Kawamura, Y., Ahrary, A., Mikuriya, Y., \& Ishii, K. (2007, April). Concept and design of a fully autonomous sewer pipe inspection mobile robot KANTARO. In Proceedings of the IEEE International Conference on Robotics and Automation (ICRA'07), Rome, Italy (pp. 136-143).

[10]. Warren, D., W. Wiers, and J. Sullins. "Pipeline inspection pig." U.S. Patent 3,786,684, issued January 22, 1974.
[11]. Krotkov, Eric. "Mobile robot localization using a single image." In Robotics and Automation, 1989. Proceedings., 1989 IEEE International Conference on, pp. 978-983. IEEE, 1989.

[12]. Wang, Yue, Eam Khwang Teoh, and Dinggang Shen. "Lane detection and tracking using B-Snake." Image and Vision computing 22, no. 4 (2004): 269-280.

[13]. Rasmussen, Christopher. "Texture-Based Vanishing Point Voting for Road Shape Estimation." In BMVC, pp. 1-10. 2004.

[14]. Rehman, Naveed Ur, and Kundan Kumar. "Implementation of an autonomous path planning \& obstacle avoidance UGV using SLAM." In Engineering and Emerging Technologies (ICEET), 2018 International Conference on, pp. 1-5. IEEE, 2018.

[15]. Valera, Antonio J. Jara, Miguel A. Zamora, and Antonio FG Skarmeta. "An architecture based on internet of things to support mobility and security in medical environments." In Consumer Communications and Networking Conference (CCNC), 2010 7th IEEE, pp. 1-5. IEEE, 2010.

[16]. Ishak, Mohamad Khairi, and Ng Mun Kit. "Design and Implementation of Robot Assisted Surgery Based on Internet of Things (IoT)." In 2017 International Conference on Advanced Computing and Applications (ACOMP), pp. 65-70. IEEE, 2017. 